\title{
Hacia una sociedad de actores globales. Las relaciones entre Europa y América Latina en el nuevo contexto global ${ }^{*}$
}

\author{
Ignacio Walker y Héctor Casanueva
}

Exponer acerca de la problemática de las relaciones entre la Unión Europea y América Latina y el Caribe en el actual contexto global es una tarea difícil, pero sin duda apasionante. En efecto, se trata de analizar una relación estratégica de varias décadas, que en la actual coyuntura global requiere redimensionarse y potenciarse de acuerdo con las nuevas realidades de una globalización llena de incertidumbres, de complejidades, pero a la vez de oportunidades.

El mapa geopolítico, geoeconómico y geoambiental del mundo ha cambiado radicalmente desde los primeros contactos birregionales, iniciados por parlamentarios de ambos lados del Atlántico en 1974, seguidos luego por las reuniones de Cancilleres en torno a cuestiones cruciales para nosotros, como la guerra civil en Centroamérica o la lucha contra las dictaduras del Cono Sur.

El apoyo europeo al Grupo de Contadora, la Paz de Esquipulas o los acuerdos de El Salvador, así como la democratización y la transición política en el Cono Sur fueron factores determinantes para que América Latina retomara una senda de institucionalización democrática. A la vez, los generosos programas de cooperación para el desarrollo y de fortalecimiento institucional han contribuido sin duda a que nuestra región alcance los niveles de crecimiento y fortaleza financiera que puede exhibir hoy en día.

Tenemos por ello una deuda de gratitud hacia la Unión Europea y en especial a los líderes de entonces, como

* Conferencia dictada por el Senador Ignacio Walker en el seminario «La dinámica de la relación LACUE en el emergente contexto internacional», organizado por el proyecto VertebrALCUE, Buenos Aires, 4-5 de junio de 2012. 
Delors, Mitterrand, Martens, Kohl, Matutes, Marín, Chaysson y tantos otros, que pusieron una mirada de largo plazo en esta relación, basados en una comunidad valórica y cultural de cinco siglos.

Entender que el mundo ha cambiado, especialmente en los últimos años, desde que en 1999 acordáramos en Río de Janeiro institucionalizar una relación birregional con carácter de "estratégica» para avanzar cualitativamente en la relación y en la construcción de un mundo multipolar, es el punto de partida de nuestra reflexión.

Junto con ello, sin embargo, debemos ser capaces de visualizar, en clave de futuro, donde está cada cual y donde deberíamos estar para construir un nuevo orden global que signifique un mayor avance en todo sentido, capaz de dar satisfacción a las necesidades materiales y espirituales de nuestros pueblos.

Porque claramente, pese a los avances, la humanidad tiene aún graves déficit de democracia, de desarrollo, de medioambiente y, en un sentido más amplio, un déficit de gobernabilidad global del que no hemos logrado aún hacernos cargo como comunidad internacional.

Seamos claros: como ha dicho Robert Gilpin, el Talón de Aquiles de la globalización es la falta de instituciones, es decir, de reglas del juego claras $\mathrm{y}$ estables (y equitativas, me atrevo a añadir). La economía y los mercados no actúan en un vacío político e institucional. Asegurar la gobernabilidad global pasa por un proceso de construcción de instituciones. Ese es el principal desafío en términos del futuro. Ese es también el desafío que enfrentamos entre Europa y América Latina.

Dentro de pocos días se realizará la Cumbre de Río+20, en la que deberemos analizar con toda franqueza si en estos veinte años hemos avanzado efectivamente en materia medioambiental y de protección de la biodiversidad.

Asimismo, tendría lugar una nueva Cumbre del G-20, grupo en el que, sumados los europeos y latinoamericanos representamos casi mil millones de personas y un porcentaje muy relevante del producto mundial.

Importante responsabilidad la que nos cabe entonces, si somos consecuentes con lo planteado en el documento de la Comisión Europea de 2009, presentado como base del diálogo en la VI Cumbre de Madrid, en cuanto a constituirnos ambas regiones en "una sociedad de actores globales».

Una sociedad de actores globales implica muchas cosas.

Desde luego, requiere de una relación igualitaria en la que ambas partes se posicionen con el mismo grado de responsabilidad, no obstante las asimetrías que existan en el tamaño de las economías o en el peso específico de cada región en el contexto global.

Implica, asimismo, una conciencia plena de la complementariedad entre ambas partes, capaz de generar sinergias políticas, productivas, financieras, ecológicas, comerciales, de mutuo beneficio. En consecuencia, ser capaces de definir los puntos focales en los que se basa esa relación estratégica, los que 
deben constituir la matriz y el sentido de la misma.

Significa, por último, entender del mismo modo el significado de «estratégico", porque muchas veces se confunde lo estratégico con lo táctico, y lo táctico con lo operativo. Entender bien esto hace la diferencia entre políticas de coyuntura y políticas de largo plazo.

En las actuales circunstancias de incertidumbres y desafíos globales, y de crisis sistémicas como la asiática de fines de los años noventa, la crisis financiera de 2008, o la actual crisis de la eurozona, debemos ser capaces de vencer la tendencia a lo puramente táctico, coyuntural y local, y situarnos en una perspectiva de largo plazo, estratégica y global.

El coyunturalismo, la mirada de corto plazo, tanto en Europa como en América Latina, tienen su expresión más nefasta en el renacer del nacionalismo, el populismo y el proteccionismo, tres elementos que atentan contra los avances de la humanidad en términos de integración, cooperación, desarrollo y paz internacional.

Recordemos que la visión de Robert Schuman, Jean Monnet, Alcides de Gasperi o Konrad Adenauer, algunos de los precursores de la Unión Europea, tuvo como enemigo declarado el nacionalismo, a partir de un sentido de la responsabilidad en la construcción de Europa, que se inicia con los acuerdos del Acero y el Carbón a fines de los años cuarenta entre Alemania y Francia, y que fue tomando forma a partir del Tratado de Roma, de 1957, todo ello en las antípodas del populismo y el proteccionismo.

Tanto en Europa como en América Latina encontramos síntomas de nacionalismo económico, de populismos de izquierdas o derechas, de miradas paranoicas y xenofóbicas frente a los procesos de inmigración, con posturas y apelando a sentimientos que cuestionan los procesos de integración, entre otros síntomas de preocupación e involución.

Este es uno de los mayores desafíos que enfrenta la relación estratégica birregional: trascender las coyunturas críticas y superar las tendencias disruptivas para avanzar hacia metas comunes en torno al diálogo político, el comercio y la cooperación, los tres pilares que ya fueron delineados con toda claridad en 1999 y ratificados sistemáticamente en las Cumbres de Madrid (2002), Guadalajara (2004), Viena (2006), Lima (2008) y Madrid (2010).

La Declaración de Madrid y el Plan de Acción 2010-2012 son actualmente la carta de navegación birregional para esta relación que hemos denominado «estratégica» desde la primera Cumbre, celebrada en Brasil en 1999.

Esperemos que en la próxima Cumbre, en Santiago, en enero de 2013, estas metas sean no solamente ratificadas sino que adquieran nuevo impulso, ya que será la primera Cumbre en que los países latinoamericanos concurriremos bajo un mismo marco político continental, en torno a la Comunidad de Estados de Latinoamérica y el Caribe (CELAC) y por ello será posible, creemos, avanzar con mayor facilidad y certeza en el en- 
tendimiento mutuo para enfrentar los desafíos comunes.

La CELAC al menos significará para la Unión Europea poder contar con un interlocutor válido y único en América Latina y el Caribe, cuestión de la que hemos carecido por años y décadas a la luz de la proliferación de iniciativas de integración regionales y subregionales, dispersas e incoherentes.

\section{EL CAMINO RECORRIDO}

Constituye un gran éxito que ininterrumpidamente, desde 1999, a contrapelo de la discontinuidad de tantas iniciativas de acercamiento, haya sido posible que las máximas autoridades de ambas regiones se reúnan a dialogar y analizar el avance de la relación, en torno a los ya mencionados componentes de Diálogo Político, Comercio y Cooperación.

El instrumento para ir construyendo la asociación estratégica y llegar al mismo tiempo a constituir una zona eurolatinoamericana de libre comercio son los «Acuerdos de Asociación», una modalidad avanzada -la más avanzada en realidad- de relacionamiento integral mediante estos tres componentes.

La idea de mediano plazo ha sido llegar a una convergencia de acuerdos que permitan una asociación birregional integral que comprenda las etapas anteriores, caracterizadas por los llamados «acuerdos de primera generación» de los años setenta, centrados fundamentalmente en la cooperación técnica, los «acuerdos de segunda generación", de los años ochenta, que incluyeron algunos componentes económicos y los «acuerdos de tercera generación» o «de cooperación avanzada», de los años noventa, en que se incorporaba un componente político y una mayor apertura al diálogo comercial, preparatorio en cierto modo de la etapa siguiente, la de los mencionados acuerdos de asociación de los decenios del 2000 y siguientes.

Es así como en la Cumbre de 1999 se anunció la negociación con Chile y México --dos países no pertenecientes a esquemas subregionales, cuyos acuerdos se encuentran plenamente vigentes-- y el anuncio de negociaciones con el Mercosur, que han sido lentas e intermitentes, y aún no finalizan.

Más tarde vinieron las negociaciones con la Comunidad Andina, que tras la salida de Venezuela han derivado en acuerdos bilaterales con Colombia y Perú, y luego con Centroamérica, todos ellos ya firmados y en proceso de ratificación.

Los resultados indican que los acuerdos con México y Chile han sido claramente beneficiosos para ambas partes, aumentando el comercio, las inversiones y la cooperación con la UE. La desgravación del universo arancelario, la reducción paulatina de las cuotas y la estabilidad de las reglas del juego, son factores fundamentales que explican estos buenos resultados.

Las proyecciones de los demás acuerdos por firmar indican que su efecto en creación de comercio y aumento 
de las inversiones también serán positivos, si bien en el caso del Mercosur el componente agrícola del comercio es un elemento de difícil tratamiento, tanto desde el punto de vista europeo -recordemos que el Parlamento Europeo, la propia Comisión, además de los grupos de productores, especialmente de Francia, han generado una fuerte oposición o condicionamiento a la negociación-, como desde el punto de vista del Mercosur, especialmente por la asimetría de los intercambios y temas sensibles como propiedad intelectual, denominaciones de origen y otras.

En la Cumbre de Madrid de 2010 se estimó que se podría llegar a la próxima Cumbre, la de Santiago, con la totalidad de los acuerdos firmados, abarcando toda la región, para iniciar así una etapa centrada en otros instrumentos. A pesar del entusiasmo inicial, no parece posible que así suceda, lo que pone una nota de cautela sobre los objetivos de la cita de Santiago en este aspecto.

Revisemos brevemente las principales áreas sobre las cuales ha discurrido la relación birregional entre Europa y América Latina y el Caribe, incluyendo el ámbito de la educación superior, la ciencia y la tecnología.

\section{DiÁlogo POLÍtico}

El diálogo político tiene lugar fundamentalmente en las Cumbres de Jefes de Estado y en las reuniones ministeriales de asuntos exteriores sobre temas estratégicos de la agenda birregional e internacional, como la pobreza y el cambio climático, la crisis económica y financiera, las energías renovables y la seguridad energética. La promoción y protección de la democracia, los derechos humanos, el derecho internacional, la paz y el derecho al desarrollo, son elementos básicos de este diálogo, declarados expresamente en el documento final de Río, en 1999.

El diálogo birregional a alto nivel se ha centrado en políticas sectoriales para el desarrollo sostenible, las drogas, la migración y el cambio climático. En el caso de los acuerdos de asociación, este componente adquiere una dimensión más bilateral.

México y Brasil son considerados formalmente por la UE como «socios estratégicos», lo que significa que son interlocutores preferentes para los temas de orden político, en una línea de consulta más permanente.

Asimismo, la creación en 2006 de la Asamblea Parlamentaria Eurolat, integrada por 75 parlamentarios de cada región, es un componente fundamental para dar mayor impulso a la relación sobre una base política más cercana a los ciudadanos.

En nuestra opinión, el componente político, que tuvo gran relevancia en épocas pasadas de consolidación democrática en América Latina, se ha visto indebidamente atenuado ante los componentes económico-comerciales.

A pesar de ello, en la Cumbre de Madrid, el diálogo de los jefes de Estado incidió en los aspectos más políticos de la crisis global y se habló de coor- 
dinar posiciones en el Grupo de los 20 , mientras que se hacía un llamado a ocuparse de los temas globales, en ámbitos prioritarios relacionados con los desafíos globales, tales como los problemas macroeconómicos y financieros, la seguridad y los derechos humanos, el empleo y los asuntos sociales, el medio ambiente, el cambio climático y la energía, la enseñanza superior y la tecnología y la innovación.

Entendemos que en la Cumbre de Santiago, que estará centrada en las inversiones de calidad y el desarrollo sustentable, habrá un espacio para que los Jefes de Estado dialoguen en torno a estas cuestiones estratégicas, para lograr una sintonía que permita contribuir en conjunto a estructurar una nueva agenda internacional en la perspectiva de una gobernabilidad global.

\section{Comercio}

En materia de comercio, para la UE somos solo el $2,3 \%$ de sus exportaciones y el 2,4\% de sus importaciones extracomunitarias. Para nosotros, sin embargo, la Unión Europea es nuestro segundo socio mundial, después de Estados Unidos, con un comercio de bienes de 174.000 millones de euros (unos 230.000 millones de dólares), y 44.000 millones de euros (unos 60.000 millones de dólares) en servicios. Ello sin perjuicio de que en una mirada de futuro, tal como advierte la CEPAL, tanto la UE como Estados Unidos podrían verse desplazados en nuestras exportaciones por China hacia mediados del presente decenio.

La balanza comercial es superavitaria para América Latina, pero debemos tener presente que la estructura del comercio es favorable a la UE, ya que por nuestra parte exportamos fundamentalmente productos básicos e importamos bienes con alto valor agregado.

La región aún no recupera el nivel de exportaciones a la UE previos a la crisis financiera de 2008, y de hecho un leve repunte producido en 2010 se ve amenazado por la crisis actual.

Puede decirse que las exportaciones tradicionales son las que más sufren esta crisis. En cambio, las no tradicionales, con valor agregado, sufren menos, porque se trata principalmente de productos de la agroindustria y alimentación, y la UE es el primer importador mundial de alimentos y lo seguirá siendo por razones de tamaño y geografía.

Razón adicional para dar una mirada de precisión a nuestras políticas de fomento productivo y desarrollo exportador, orientadas al mercado europeo.

\section{INVERSIONES}

El estrechamiento de los vínculos ha repercutido también de manera notable en las inversiones y el comercio.

En efecto, para América Latina la UE es la primera fuente mundial de inversiones y representa el $43 \%$ de los flujos llegados a la región. España representa casi el $50 \%$ y le siguen el Reino Unido, Francia y Alemania. 
En la última década, las inversiones europeas en la región han alcanzado en promedio 30 mil millones de dólares anuales y, en total, la inversión europea acumulada históricamente se eleva a 465 mil millones de dólares, que supera la inversión hecha en China, India y Rusia en conjunto.

Sin contar los flujos netamente financieros, que van mayoritariamente son Brasil $(40 \%)$, México (25\%), Argentina $(15 \%)$ y Chile $(6 \%)$ hacia el Caribe, los países concentran las inversiones productivas y en servicios, en particular en los sectores de energía, telecomunicaciones, recursos naturales, y servicios financieros.

Un dato muy relevante: el Mercosur concentra 165 mil millones de dólares de esta inversión. Es de esperar que el actual diferendo con Argentina sobre REPSOL no signifique una merma en las intenciones de invertir de las empresas comunitarias Afortunadamente, en los últimos días hemos visto algunas declaraciones de las autoridades comunitarias y del propio ministro español de asuntos exteriores, que apuntan al diálogo y a la búsqueda de soluciones razonables.

\section{COOPERACIÓN}

Fruto de la asociación estratégica, en estos años han surgido importantes programas de cooperación, en torno a los ejes de cohesión social, integración regional, educación superior, inversiones y comprensión mutua.
De estos ejes han derivado importantes programas en ámbitos como la cooperación entre universidades europeas y latinoamericanas (ALFA), el intercambio de estudiantes (ERASMUS MUNDUS), la promoción de negocios y la internacionalización de las Pymes (AL-INVEST), la cooperación para el desarrollo urbano entre ciudades (URB-AL); la sociedad de la información (@LIS); la cooperación en materia de salud, justicia, educación, empleo para el apoyo a la cohesión social (EUROSOCIAL) y el cambio climático (EUROCIIMA).

A partir de la Cumbre de Madrid de 2010, hemos visto los programas LAIF para apalancar inversiones destinadas a la integración territorial, la conectividad, la protección del medio ambiente y el desarrollo productivo de las pymes, el programa EUROSOLAR para promover el desarrollo de la energía solar; el programa COPOLAD en materia de drogas y la Red RALCEA para la realización de estudios sobre el agua.

Los programas anteriores y en general la cooperación comunitaria a la región a través de 450 programas y proyectos representan entre 2007 y 2013, un aporte de 3 mil millones de euros (unos 4.500 millones de dólares), lo que significa que la Unión Europea es, por lejos, el primer cooperante mundial en la región. También lo es, por cierto, a nivel mundial, ya que la UE representa el $50 \%$ del total de la asistencia mundial para el desarrollo.

A lo anterior hay que agregar lo acordado en Madrid hace dos años 
en cuanto a que el Banco Europeo de Inversiones (BEI) está autorizado para otorgar préstamos a América Latina hasta por 2.800 millones de euros para inversiones en infraestructura. Los proyectos de infraestructura de la integración, de los programas IIRSA y Mesoamérica, se verán sin duda beneficiados con la cooperación del BEI.

\section{LA EDUCACIÓN SUPERIOR, LA CIENCIA Y LA TECNOLOGÍA EN LA RELACIÓN UE-ALC}

Estamos en un encuentro de universidades y por ello me referiré al componente de educación superior y de ciencia y tecnología en nuestras relaciones. Lo haré muy brevemente, ya que en el desarrollo del seminario se abordaran estas áreas con mayor especialización y profundidad.

En todo el mundo la educación superior es objeto de grandes transformaciones y desafíos en materia de acceso, financiamiento, diversidad y calidad, producto del crecimiento de estratos medios, que particularmente en América Latina ha sido muy dinámico en los últimos decenios, en especial en Brasil, Chile, Colombia, y el Perú.

Pese a su heterogeneidad, América Latina ya no es una región que pueda identificar como la de países subdesarrollados, del Tercer Mundo, que conocimos en los años setenta sino como países de ingreso medio. Los cambios demográficos y en el campo de la educación, asociados al fenómeno de la movilidad social, están en el centro de esos procesos.

Las universidades deben, responder con mayor eficiencia al reto de la competitividad global, pero sin dejar de lado su esencia de búsqueda de la verdad y transmisión del conocimiento.

Mientras en la UE se trabaja en la armonización de las carreras universitarias y la movilidad estudiantil, por ejemplo, mediante el proceso de Bolonia y otros programas, en América Latina debemos responder al reto de la inclusión y la cohesión social, especialmente tomando en cuenta la creciente demanda de los jóvenes de sectores emergentes y aspiracionales. Al respecto, un dato:

En América Latina, el número de estudiantes en la educación superior pasó de 276 mil en 1950 a más de 15 millones en la actualidad, con una oferta de más de 3 mil instituciones, la mayoría de ellas privadas. Esto produce una enorme diversidad y desniveles de calidad y equidad que deben ser abordados y resueltos. De ahí que la cooperación entre ambas regiones en esta área sea fundamental, tanto para la transmisión de experiencias como mejorar los procesos educativos, la formación de excelencia y las sinergias que ello produce para beneficio mutuo.

Debo decir que, con mucha visión, ya la primera Cumbre birregional se ocupó de establecer como una de los proyectos prioritarios, la educación en general y la ciencia y la tecnología en particular. Junto con ocuparse en varios de sus acápites de este tema, la 
Declaración de los Jefes de Estado y de Gobierno alude expresamente, en su punto 63, a la cooperación entre las universidades, y en sus puntos 65 y 66, al impulso a la investigación científica y la innovación. Todo ello, con el fin de utilizar los recursos humanos para el desarrollo integral de ambas regiones.

Estos propósitos se fueron reiterando sucesivamente en las Cumbres siguientes y han dado origen a programas muy relevantes.

A partir de la Cumbre de Guadalajara, de 2004, se da prioridad a la consolidación de un espacio común de educación superior entre las dos regiones. En este contexto uno de los propósitos de la Comisión Europea, ha sido recibir a más de 4 mil estudiantes y profesores latinoamericanos en las universidades europeas en el período 2007-2013.

A modo de ejemplo, cabe señalar que la cooperación en materia de educación superior, promovida mediante los programas ALFA I, II y III, ha significado hasta el momento el apoyo a 1.070 proyectos, de 700 universidades, con un aporte comunitario de 55 millones de euros (unos 67 millones de dólares). Por su parte, durante los 5 años de convocatorias el programa de becas ALBAN seleccionó tres mil 319 candidatos de 18 países de América Latina para becas de estudios de Maestrías, Doctorados o especializaciones avanzadas en diversas instituciones de los Estados miembro de la Unión Europea.
Asimismo, en lo que respecta a ciencia y tecnología y promoción de la sociedad de la información y del espacio eurolatinoamericano del conocimiento, el instrumento más importante en volumen e impacto han sido los Programas marco de ciencia y tecnología de la UE extendidos hacia América Latina y que, a su vez, dan origen a otros programas y redes, como EURALINET o el programa de interconexión avanzada ALICE II generado desde la Red Clara de interconexión latinoamericana y europea.

Los Programas marco de Ciencia y Tecnología se extendieron a América Latina a partir del año 2000 y cuentan con aportes de ambas partes. Por ejemplo, el VI Programa marco significó 221 proyectos conjuntos de universidades, con la participación de 538 investigadores de América Latina, mientras que el VII PM, vigente hasta 2013, ya cuenta con más de 600 investigadores de la región.

Justamente por la importancia de la cooperación en educación superior, un grupo de universidades chilenas, en conjunto con más de cien universidades de la UE y América Latina -seguramente varias de ellas presentes en esta salaestán organizando para enero de 2013 una «Cumbre Académica UE-ALC» paralela a la Cumbre CELAC-UE, en la que se debatirán las proyecciones del espacio común de educación superior, los lineamientos del próximo Programa marco de c y t de la UE que debería iniciarse en 2014 y el futuro de la relación estratégica birregional. 
El futuro de la Asociación ESTRATÉGICA UNIÓN EUROPEAAmérica latina y el Caribe.

Para muchos, la situación actual de Europa no favorece una mirada optimista respecto del futuro de las relaciones con América Latina y el Caribe. Analistas de ambas regiones siembran el escepticismo o son directamente agoreros del desastre en el caso de la Unión Europea y promotores de un cambio societario en el caso de América Latina, en el sentido de que debiéramos poner el énfasis estratégico en el Asia --en China en particular-- y en Estados Unidos, en lugar de Europa. Son miradas simplistas, que siempre han existido, pero que ahora adquieren mayor visibilidad y relevancia dadas las circunstancias por la que atraviesa el Viejo Continente.

Sin embargo, lo expuesto hasta aquí en cuanto a comercio, inversiones, cooperación y sintonía política entre ambas regiones demuestra que en la difícil coyuntura europea hay elementos sólidos y de largo plazo, así como desafíos comunes de carácter estratégico, que ameritan una apuesta por esa «sociedad de actores globales» que señalaba la Comisión Europea en 2009.

Por una parte, el peso específico de la UE, que sigue siendo la primera potencia comercial del mundo el $28 \%$ del PIB mundial, con solo el $7 \%$ de la población; el primer importador mundial de alimentos y pese a los fuertes recortes en este ámbito la primera fuente de cooperación para el desarrollo, paradójicamente sigue siendo una fuente de inversiones potente, ya que los excedentes de sus empresas les permiten buscar espacios de expansión en los cuales posicionarse.

No es un dato menor que en la propuesta presupuestaria de la UE para 2014-2020, consistente con el Plan Europa 2020 , se prevea un aumento del $46 \%$ en el campo de la ciencia y la tecnología para apoyar su competitividad global.

Por otra parte, América Latina se encuentra en la mejor posición de su historia, con sistemas democráticos y un desarrollo institucional avanzado, estabilidad macroeconómica y financiera, crecimiento económico, auge exportador e incremento de su peso político global, especialmente por la incorporación al G-20 de tres países de la región y el hecho de que cuenta con una población joven, recursos naturales y la biodiversidad que el mundo requiere. Lo anterior, no obstante que representa solo el $8 \%$ del PIB mundial, con déficit y asimetrías, principalmente en el nivel del desarrollo social.

Estas nuevas circunstancias, en medio de la crisis de proporciones por la que atraviesa Europa, con las complejidades institucionales de un proceso de integración aún no consolidado totalmente y, por otra parte, una América Latina emergente capaz de desempeñar un rol global, pueden llegar a configurar, como lo señalan importantes analistas internacionales, avalados por datos de la CEPAL, una oportunidad para complementarse y generar condiciones en las que de esta unión surja la fuerza que Europa 
necesita para trascender la coyuntura y que América Latina requiere para consolidar su proceso de desarrollo incorporando conocimientos, ciencia, tecnología y oportunidades de mercado.

Al mismo tiempo, en el plano político global, la nueva arquitectura financiera y de gobernabilidad global requiere de la sinergia de ambas regiones, que pueden, si se lo proponen con auténtica voluntad política, aliarse en torno a cuestiones claves como la conclusión de la Ronda de Doha, la reforma del sistema financiero y la lucha en el ámbito del cambio climático.

Creemos que en la próxima cita birregional, que será la Primera Cumbre de CELAC-UE, esta mirada debería estar presente más allá de dar continuidad a programas y proyectos vigentes y rectificar y enmendar rumbos en aquello que así lo amerite.

Reunir en Santiago a sesenta jefes de Estado y de gobierno, que representan mil millones de personas y casi el $40 \%$ del PIB mundial en la actual coyuntura internacional y en medio de las demandas crecientes por mayor desarrollo social, oportunidades y certidumbres para los jóvenes es una ocasión para dar una señal política al mundo en el sentido que las personas esperan, en el marco de una sociedad de actores globales.

Se trata por tanto no solamente de mejorar las condiciones en que nos relacionamos y crecemos, sino también de asumir una responsabilidad histórica de que ambas regiones contribuyan unidas a construir un mundo mejor. 